\title{
Avaliação da qualidade das córneas doadoras em relação à idade do doador e causa do óbito
}

\author{
Evaluation of the quality of donor corneas in relation to the age \\ of donor and cause of death
}

\author{
Gleisson Rezende Pantaleão ${ }^{1}$ \\ Márcio Zapparolli² \\ Guilherme Barroso Guedes $^{3}$ \\ Wilson Moreira Dimartini Junior ${ }^{4}$ \\ Cristiane Carvalho Vidal ${ }^{5}$ \\ Daniel Wasilewski ${ }^{6}$ \\ Hamilton Moreira ${ }^{7}$
}

Trabalho realizado no Banco de Tecidos Oculares do HC-UFPR - Curitiba (PR) - Brasil.

${ }^{1}$ Estagiário de Segmento Anterior do Banco de Tecidos Oculares do Hospital de Clínicas da Universidade Federal do Paraná - UFPR - Curitiba (PR) - Brasil.

${ }^{2}$ Estagiário de Segmento Anterior do Banco de Tecidos Oculares do Hospital de Clínicas da UFPR - Curitiba (PR) - Brasil.

${ }^{3}$ Residente em Oftalmologia do Hospital de Clínicas da UFPR - Curitiba (PR) - Brasil.

${ }^{4}$ Matemático, Residente em Oftalmologia do Hospital de Clínicas da UFPR - Curitiba (PR) - Brasil.

5 Acadêmica do terceiro ano de Enfermagem das Faculdades Pequeno Príncipe - Curitiba (PR) - Brasil; Estagiária em captação de córneas do Banco de Tecidos Oculares do Hospital de Clínicas da UFPR - Curitiba (PR) - Brasil. ${ }^{6}$ Responsável Técnico substituto do Banco de Tecidos Oculares do Hospital de Clínicas da UFPR - Curitiba (PR) - Brasil.

${ }^{7}$ Doutor, Professor Adjunto, Responsável Técnico do Banco de Tecidos Oculares do Hospital de Clínicas da UFPR - Curitiba (PR) - Brasil; Professor Assistente da Faculdade Evangélica do Paraná - Curitiba (PR) - Brasil. Endereço para correspondência: Hamilton Moreira. Rua Coronel Dulcídio, 199 - 4 andar - Curitiba (PR) CEP 80420-170

E-mail: gleissonrp@hotmail.com hamiltonmoreira@onda.com.br

Recebido para publicação em 11.02.2009

Versão recebida em 03.08.2009

Aprovação em 04.08.2009

\begin{tabular}{|c|}
\hline RESUMO \\
\hline $\begin{array}{l}\text { Objetivo: Analisar a qualidade das córneas avaliadas em lâmpada de } \\
\text { fenda no Banco de Tecidos Oculares do Hospital de Clínicas da Univer- } \\
\text { sidade Federal do Paraná (BTO HC-UFPR) relacionando com a idade e } \\
\text { causa de óbito. Métodos: Foram analisadas retrospectivamente fichas de } \\
\text { avaliações padronizadas de } 492 \text { córneas, avaliadas no BTO HC-UFPR, do } \\
\text { período de agosto de } 2006 \text { a agosto de } 2008 \text {. Cada córnea avaliada foi } \\
\text { classificada em relação a sua qualidade em: Muito bom, bom, regular e } \\
\text { ruim; mediante os seguintes fatores: claridade da córnea, edema epitelial, } \\
\text { defeito epitelial, edema estromal, dobras de Descemet, opacidade estromal, } \\
\text { cicatrizes corneanas, densidade endotelial e guttata. Resultados: A idade } \\
\text { média dos doadores foi de } 42,74 \text { anos (dp=17,77). Dentre as causas de } \\
\text { óbito, a mais comum foi trauma com } 46,18 \% \text {, seguindo por causas car- } \\
\text { diovasculares com } 41,86 \% \text { Na avaliação da córnea, foram classificadas: } \\
57,11 \% \text { como "bom", seguidopor } 20,73 \% \text { "regular", } 16,87 \% \text { "muito bom" } \\
\text { e } 5,28 \% \text { "ruim". Com aplicação de testes estatísticos baseados em } \\
\text { modelo de regressão ordinal verificou-se que as córneas provenientes de } \\
\text { mortes por traumas tendem a ser melhores, bem como aquelas de pa- } \\
\text { cientes mais jovens. Conclusões: Estatisticamente, córneas de doadores } \\
\text { mais jovens tendem a ter melhores graduações na avaliação, bem como } \\
\text { córneas provenientes de doadores com morte por trauma tendem a ser } \\
\text { de melhor qualidade quando comparadas com as provenientes de mortes } \\
\text { por doenças cardiovasculares e outras doenças sistêmicas. }\end{array}$ \\
\hline
\end{tabular}

Descritores: Transplante de córnea; Doadores de tecidos; Banco de tecidos; Controle de qualidade

\section{INTRODUÇÃO}

O banco de tecidos oculares (BTO) tem a função de captar, avaliar, preservar e distribuir córneas e outros tecidos oculares, sendo responsabilidade deste, assegurar que as córneas apresentem padrão satisfatório aos cirurgiões transplantadores ${ }^{(1)}$.

Com o aumento do número de córneas doadas e armazenadas por períodos mais longos, a avaliação criteriosa torna-se procedimento indispensável e de grande responsabilidade dos bancos de tecidos oculares. Com este enfoque, alguns dados sobre a córnea doadora como a idade do doador e causa do óbito, são pautas de dúvidas sobre a qualidade da córnea ${ }^{(1-2)}$.

Alguns trabalhos referem a idade da córnea doadora como não sendo fator determinante na qualidade da córnea ${ }^{(3-5)}$.

O objetivo deste trabalho foi avaliar a idade e causa de óbito em relação a qualidade das córneas avaliadas em lâmpada de fenda no Banco de Tecidos 
Oculares do Hospital de Clínicas da Universidade Federal do Paraná (BTO HC-UFPR) com intuito de melhorar e garantir a qualidade das córneas distribuídas.

\section{MÉTODOS}

Foram analisadas retrospectivamente, fichas de avaliação de córnea em lâmpada de fenda de 492 córneas doadoras avaliadas no período de agosto de 2006 a agosto de 2008 no BTO HCUFPR. As córneas foram submetidas à avaliação na lâmpada de fenda com aumento de 40 vezes e a preservação feita em meio de conservação Optisol GS.

A avaliação, que segue normas internacionais do EBAA (Eye Bank Association of América), é feita por oftalmologistas estagiários em cirurgias do segmento anterior e transplante de córnea do BTO HC-UFPR, que foram treinados em curso específico para tal função ${ }^{(6)}$.

Os fatores analisados e anotados na ficha de avaliação padronizada foram: claridade da córnea, edema epitelial, defeito epitelial, edema estromal, dobras na Descemet, opacidade estromal, cicatrizes corneanas, perda de células endotelias e gutatta. Após avaliação, é dada uma classificação geral que varia de 1 a 4, sendo: muito bom (1), bom (2), regular (3) e ruim (4). Esta classificação foi baseada na pior classificação individual dos itens citados anteriormente. Por exemplo, se uma córnea tinha vários itens classificados como "muito bom" e apenas 1 item classificado com "ruim", ela foi classificada no quadro geral como "ruim".

As córneas foram separadas por grupos de idade do doador, sendo: de 5 a 20 anos, de 21 a 35, de 36 a 50, de 51 a 65, de 66 a 80 e maiores de 80 anos. Outra classificação foi quanto à causa de óbito dos doadores, sendo divididos em: trauma (arma de fogo, acidentes automobilísticos, traumatismo craniano e outros), doenças cardiovasculares, outras doenças sistêmicas, doenças neoplásicas e causas indeterminadas.

A captação das córneas pelo BTO HC-UFPR é feita por técnicos de enfermagem e médicos residentes em oftalmologia devidamente treinados pelos responsáveis técnicos do banco.

O processo de captação de córneas no BTO HC-UFPR é feita por meio de retirada "in situ", ou seja, apenas a córnea e anel córneo-escleral de cerca de $4 \mathrm{~mm}$ é captado, sendo o doador recomposto esteticamente após o procedimento. O tempo permitido entre o óbito e a retirada da córnea no BTO HC-UFPR é de no máximo 12 horas, portanto levando em consideração o processo de retirada da córnea e a preservação em meio Optisol GS, o tempo de preservação foi de no máximo 14 horas após o óbito. A causa da morte foi obtida pela ficha preenchida durante a captação e esta por sua vez era anotada seguindo o atestado de óbito ou documento fornecido pelo hospital.

Após coleta de dados, foram feitas correlações entre a qualidade da córnea segundo avaliação em lâmpada de fenda com a idade do doador e causa de óbito.

Para a análise dos dados foram utilizados os programas: $\mathrm{R}$ [R: Copyright 2005, The R Foundation for Statistical Compu- ting, Version 2.0.1 Patched (2005-03-30), ISBN 3-900051-07-0], o programa SPSS for Windows versão 10.0.1 e Excel for Windows 2007. O intervalo de confiança adotado foi de $95 \%$ e significância de 5\%. Foi realizado análise dos dados baseados num modelo de regressão ordinal, com teste qui-quadrado e função de ligação "Loglog".

\section{RESULTADOS}

A amostra analisada foi composta de 492 córneas de 275 doadores, com média de idade de 42,74 anos $(\mathrm{dp}=17,77)$ e variou de 7 a 85 anos. A distribuição por faixa etária foi: 40 (14,55\%) entre 5 e 20 anos de idade, $71(25,82 \%)$ com idade entre 21 e 35 anos, $55(20,00 \%)$ entre 36 e 50 anos, $77(28,00 \%)$ entre 51 e 60 anos, $30(10,91 \%)$ entre 61 e 80 anos e $2(0,73 \%)$ com idade acima de 80 anos (Gráfico 1).

Quanto à causa do óbito, a mais prevalente foi trauma com 127 (46,18\%), seguido por cardiovasculares com 115 óbitos $(41,86 \%)$, outras doenças sistêmicas com 17 (6,18\%), indeterminadas com $11(4,00 \%)$ e neoplasias com $5(1,82 \%)$ (Gráfico 2).Quanto à classificação da qualidade da córnea durante a avaliação em lâmpada de fenda; das 492 córneas avaliadas encontramos classificação como "bom" em 281 $(57,11 \%), 83(16,87 \%)$ como “muito bom”, $102(20,73 \%)$ como

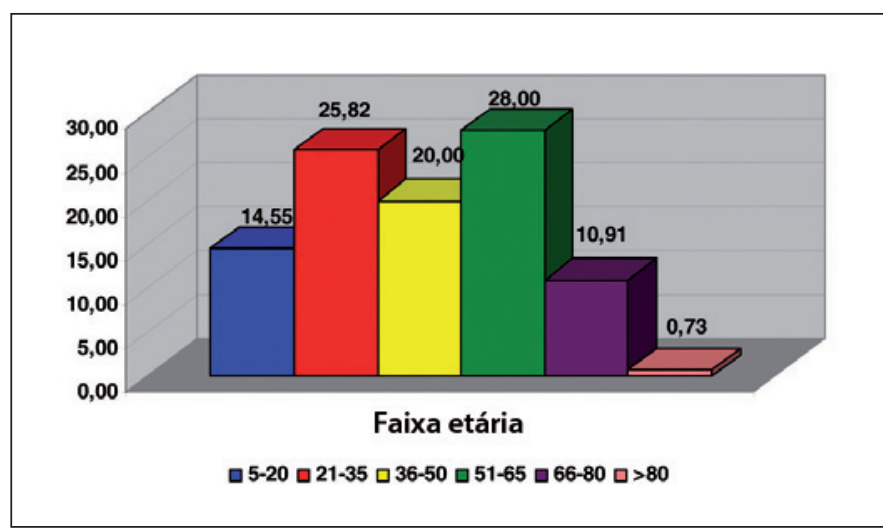

Gráfico 1 - Distribuição porcentual dos doadores por faixa etária

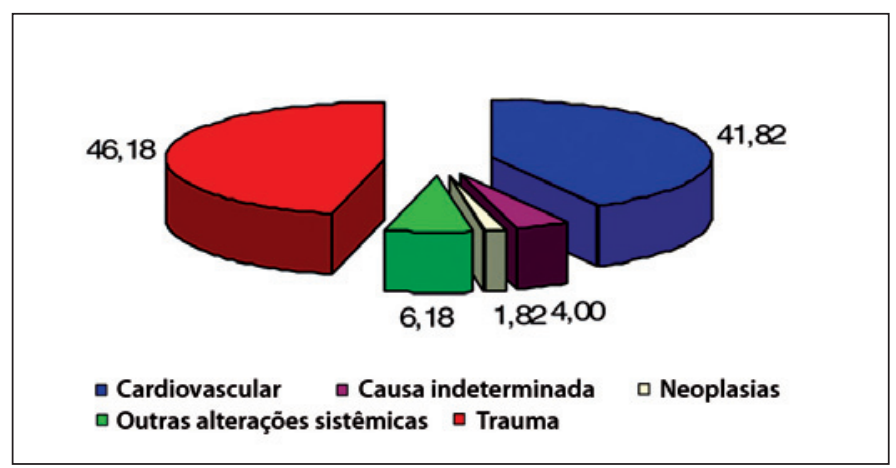

Gráfico 2 - Distribuição porcentual de óbitos por causa 
"regular" e 26 (5,28\%) como "ruim"; como é possível verificar no gráfico 3 .

Com estes dados coletados, verificamos a associação entre as características das córneas doadoras (idade do doador e a causa de óbitos), e comparamos com a classificação de qualidade na avaliação da córnea, como se observa nas tabelas.

Representamos na tabela 1 a avaliação da associação entre causa de óbito e a classificação de qualidade em lâmpada de fenda. Os testes estatísticos não puderam ser aplicados por causa do pequeno número de córneas nos grupos de óbito por causa indeterminada e neoplasias.

$\mathrm{Na}$ tabela 2, representamos a associação entre as diversas faixas etárias dos doadores com sua classificação da córnea. Com analise dos dados coletados e aplicação de teste estatístico baseado em um modelo de regressão ordinal, existem evidências estatísticas de que córneas em faixas etárias mais altas, como as de 66-80 e acima de 80 anos, têm maiores chances de apresentar avaliação de qualidade inferior às córneas de faixas etárias menores.

Após excluirmos os grupos de causas indeterminadas e neoplasias, foi possível a aplicação de testes estatísticos baseados em modelo de regressão ordinal e foi verificado que morte por causas cardiovasculares parecem influenciar de forma negativa na qualidade da córnea. Ou seja, as córneas doadoras provenientes de óbitos por trauma têm maiores chances de terem uma avaliação de melhor qualidade.

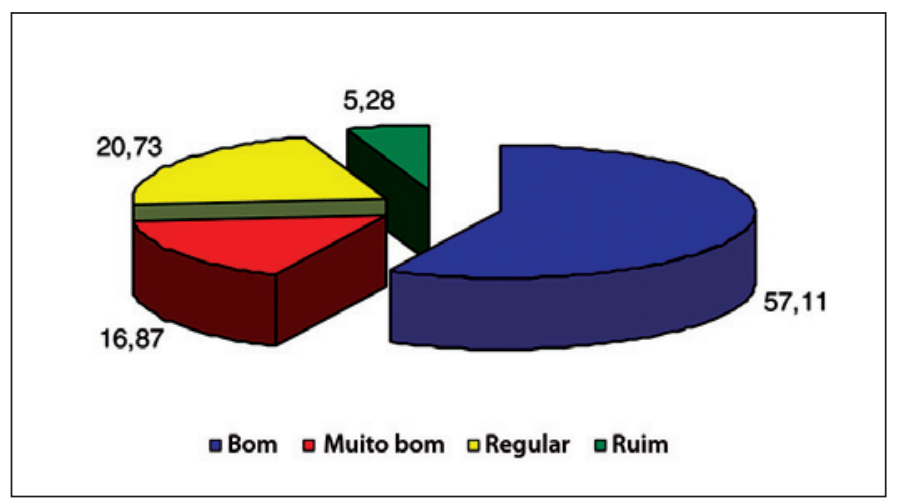

Gráfico 3 - Porcentual geral de classificação das córneas avaliadas
Estes dados podem ser certificados estatisticamente como observamos na tabela $3 \mathrm{~A}$, onde o valor de "estimate" quanto maior for, significa uma melhor qualidade da córnea; e a coluna "sig" refere-se à significância do teste.

Analogamente ao explicado anteriormente, na tabela 3B, temos os testes de regressão ordinal que mostra estatisticamente que quando mais novo o doador, melhor a córnea. A faixa etária de maiores de 80 anos foi excluída por causa do baixo número de córneas.

Na tabela 4, relacionou-se cada faixa etária com a causa de óbito, evidenciando que: as córneas provenientes de mortes por trauma tendem a serem de doadores mais jovens (sendo 86,04\% menores de 50 anos); enquanto que as córneas provenientes de mortes por doenças cardiovasculares tendem a serem provenientes de doadores mais idosos (apenas 40,28\% são menores de 50 anos).

\section{DISCUSSÃO}

As avaliações de córnea do Banco de Tecidos Oculares do Hospital de Clínicas da UFPR segue padrões internacionais como os aplicados em outros estudos ${ }^{(1-2,7)}$.

A média de idade dos doadores no estudo foi de 42,74 anos $(\mathrm{dp}=17,77)$, ficando abaixo do encontrado em outros estudos. Farias et al. encontrou uma média de 55 anos ( $\mathrm{dp}=14,8$ anos) e Georgiadis et al. encontrou média de 52 $\operatorname{anos}^{(1,8)}$. O BTO HC-UFPR faz captação em diversos hospitais da região metropolitana de Curitiba, sendo que as principais instituições que contribuem nos chamados da equipe de captação são aquelas referências para atendimento a politraumatizados, o que poderia justificar uma média de idade menor que a encontrada em outros estudos, uma vez que os indivíduos sujeitos a trauma são de menores faixas etárias que aqueles que sofrem óbito por causas naturais ou enfermidades.

A literatura científica mostra que não existe limite de idade para córneas doadoras e a idade não é fator de baixa sobrevivência do enxerto; e sim o que deve ser levado em consideração na utilização das córneas é a qualidade pela avaliação biomicroscópia e de microscopia especular do endotélio ${ }^{(3,5)}$. Por

\begin{tabular}{|c|c|c|c|c|c|c|}
\hline \multirow[b]{2}{*}{ Classificação } & \multicolumn{6}{|c|}{ Causa da morte (porcentual pela causa de óbito) } \\
\hline & Cardiovascular & $\begin{array}{c}\text { Causa } \\
\text { indeterminada }\end{array}$ & Neoplasias & $\begin{array}{c}\text { Outras } \\
\text { alterações } \\
\text { sistemicas }\end{array}$ & Trauma & Total geral \\
\hline Muito bom & $5,19 \% \quad(11)$ & $0,00 \%$ & $0,00 \%$ & $13,79 \%(4)$ & $30,63 \%(68)$ & $16,87 \%(281)$ \\
\hline Bom & $53,30 \% \quad(113)$ & $100,00 \%(20)$ & $100,00 \% \quad(9)$ & $44,83 \%(13)$ & $56,76 \% \quad(126)$ & $57,11 \%(83)$ \\
\hline Regular & $34,43 \%(73)$ & $0,00 \%$ & $0,00 \%$ & $41,38 \%(12)$ & $7,66 \%(17)$ & $20,73 \%(102)$ \\
\hline Ruim & $7,08 \%(15)$ & $0,00 \%$ & $0,00 \%$ & $0,00 \%$ & $4,95 \%(11)$ & $5,28 \%(26)$ \\
\hline Total geral & $100,00 \% \quad(212)$ & $100,00 \%(20)$ & $100,00 \%$ & $100,00 \%$ & $100,00 \% \quad(222)$ & $100,00 \%$ \\
\hline
\end{tabular}




\begin{tabular}{|c|c|c|c|c|c|c|c|}
\hline \multirow[b]{2}{*}{ Classificação } & \multicolumn{6}{|c|}{ Distribuição por faixa etária } & \multirow[b]{2}{*}{ Total geral } \\
\hline & $5-20$ & $21-35$ & $36-50$ & $51-65$ & $66-80$ & $>80$ & \\
\hline Muito bom & $45,83 \% \quad(33)$ & $25,20 \% \quad(31)$ & $16,35 \%(17)$ & $1,43 \% \quad(\quad 2)$ & $0,00 \%$ & $0,00 \%$ & $16,87 \%(83)$ \\
\hline Bom & $54,17 \%$ (39) & $60,98 \% \quad(75)$ & $53,85 \%(56)$ & $57,14 \% \quad(80)$ & $60,78 \% \quad(31)$ & $0,00 \%$ & $57,11 \%(281)$ \\
\hline Regular & $0,00 \%$ & $12,20 \%(15)$ & $20,19 \%(21)$ & $35,00 \% \quad(49)$ & $29,41 \% \quad(15)$ & $100,00 \%(2)$ & $20,73 \%(102)$ \\
\hline Ruim & $0,00 \%$ & $1,63 \% \quad(\quad 2)$ & $9,62 \% \quad(10)$ & $6,43 \% \quad(9)$ & $9,80 \% \quad(5)$ & $0,00 \%$ & $5,28 \%(26)$ \\
\hline Total geral & $100,00 \%(72)$ & $100,00 \% \quad(123)$ & $100,00 \% \quad(104)$ & $100,00 \% \quad(140)$ & $100,00 \% \quad(51)$ & $100,00 \%(2)$ & $100,00 \%$ \\
\hline
\end{tabular}

Tabela 3. Testes estatísticos

\begin{tabular}{|c|c|c|}
\hline Causa de óbito & Estimate & Sig \\
\hline $\begin{array}{l}\text { Doenças } \\
\quad \text { cardiovasculares }\end{array}$ & $-1,661$ & 0,049 \\
\hline $\begin{array}{l}\text { Outras alterações } \\
\text { sistêmicas }\end{array}$ & 0,137 & 0,869 \\
\hline Trauma & 2,362 & 0,005 \\
\hline
\end{tabular}

\begin{tabular}{|c|c|c|}
\hline Faixa etária & Estimate & Sig \\
\hline $5-20$ & 2,748 & 0,001 \\
\hline 21-35 & 2,174 & 0,010 \\
\hline $36-50$ & 2,026 & 0,015 \\
\hline $51-65$ & 1,215 & 0,143 \\
\hline $66-80$ & 1,022 & 0,226 \\
\hline
\end{tabular}

\begin{tabular}{|c|c|c|c|c|c|}
\hline $\begin{array}{l}\text { Faixa } \\
\text { etária }\end{array}$ & Cardiovascular & Trauma & Neoplasias & $\begin{array}{l}\text { Outras } \\
\text { alterações sistêmicas }\end{array}$ & $\begin{array}{c}\text { Causa } \\
\text { indeterminada }\end{array}$ \\
\hline $5-20$ & $2,84 \%(6)$ & $26,58 \%(59)$ & $11,11 \%(1)$ & $20,69 \%(6)$ & $0,00 \%$ \\
\hline 21-35 & $9,95 \% \quad(21)$ & $42,34 \% \quad(94)$ & $0,00 \%$ & $13,79 \%$ ( 4$)$ & $21,05 \%(4)$ \\
\hline $36-50$ & $27,49 \% \quad(58)$ & $17,12 \% \quad(38)$ & $0,00 \%$ & $27,59 \% \quad(8)$ & $0,00 \%$ \\
\hline $51-65$ & $47,39 \% \quad(100)$ & $10,81 \%(24)$ & $44,44 \%(4)$ & $13,79 \%(4)$ & $36,84 \%(7)$ \\
\hline $66-80$ & $11,37 \%(25)$ & $3,15 \% \quad(\quad 7)$ & $44,44 \%(4)$ & $24,14 \%$ ( 7$)$ & $42,11 \%(8)$ \\
\hline$>80$ & $0,95 \%(2)$ & $0,00 \% \quad(\quad 0)$ & $0,00 \%$ & $0,00 \%$ & $0,00 \%$ \\
\hline Total & $100,00 \% \quad(212)$ & $100,00 \% \quad(222)$ & $100,00 \%(9)$ & $100,00 \%$ & $100,00 \%$ \\
\hline
\end{tabular}

isso é importante uma avaliação criteriosa durante biomicroscopia da córnea doadora.

No presente estudo verificamos uma maior probabilidade de córneas de faixa etária mais jovem apresenta uma melhor classificação quanto a qualidade. Este dado também foi verificado em outros estudos como o de Farias et al. que mostrou que córneas de doadores acima de 60 anos têm piores avaliações quando comparados aos menores de 60 anos, o que também foi verificado por Gain et al ${ }^{(1,7)}$.

Krohn et al. conclui em seu estudo que doenças graves e debilitantes (doenças sistêmicas), geram caquexia e aumento do catabolismo que tem como consequência uma redução do número de células corneanas endoteliais, diferentemente de óbitos com morte rápida como traumas ${ }^{(9)}$. Isso explica os dados encontrados no presente estudo, onde verificamos uma pior classificação nas faixas etárias mais altas e nos óbitos por causas cardiovasculares e outras alterações sistêmicas.
Além disso, estes dois dados (alta faixa etária e presença de doenças debilitantes) tendem a coexistir e aumentam as chances de encontrarmos uma pior avaliação de qualidade quando comparamos com córneas de doadores jovens e com mortes por trauma.

\section{CONCLUSÕES}

As córneas de faixas etárias maiores como os maiores de 65 anos e aquelas provenientes de doadores vítimas de óbitos por doenças sistêmicas e debilitantes tendem a ser de pior qualidade quando comparados às baixas faixas etárias e mortes por trauma. Entretanto, esses dados não podem ser considerados isoladamente pelos transplantadores para aceitar ou rejeitar uma córnea e sim deve ser verificada a qualidade da córnea registrada pelo exame biomicroscópio minucioso. 


\section{ABSTRACT}

Purpose: To analyze the quality of the corneas evaluated by slit lamp examination in the Eye Bank of the Hospital de Clínicas UFPR and its relation to donor age and cause of death. Methods: Analysis of 492 corneas, evaluated in BTO HCUFPR between August 2006 to August 2008. Each cornea was classified regarding the quality as: very good, good, regular and bad; and according to clarity, epithelial defect, stromal edema, Descemet's folds, stromal opacity, corneal scarring, endothelial density and guttata. Results: The mean donor age was 42.74 years ( $\mathrm{sd}=17.77$ years). Among the causes of death, trauma was the most common with $46.18 \%$, followed by cardiovascular causes with $41.86 \%$. In relation to the cornea, $57.11 \%$ were classified as "good", followed by $20.73 \%$ as "regular", $16.87 \%$ as "very good" and $5.28 \%$ as "bad". With the application of statistical tests based on ordinal regression model, trauma deaths corneas tend to be better, as well as those of younger patients. Conclusions: Statistically, the corneas from younger donors tend to have better graduations in the assessment, as well as the corneas from donors dead by trauma that tend to have better quality when compared to corneas from donors dead by cardiovascular and other systemic diseases.

Keywords: Corneal transplantation; Tissue donors; Tissue bank; Quality control

\section{REFERÊNCIAS}

1. Farias RJM, Kubokawa KM, Schirmer M, Sousa LB. Avaliação de córneas doadoras em lâmpada de fenda e microscopia especular durante o período de armazenamento. Arq Bras Oftalmol. 2007;70(1):79-83.

2. Lass JH, Gal RL, Ruedy KJ, Benetz BA, Beck RW, Baratz KH, Holland EJ, Kalajian A, Kollman C, Manning FJ, Mannis MJ, McCoy K, Montoya M, Stulting D, Xing D; Cornea Donor Study Group. An evaluation of image quality and accuracy of eye bank measurement of donor cornea endothelial cell density in the Specular Microscopy Ancillary Study. Ophthalmology. 2005;112(3):431-40. Erratum in: Ophthalmology. 2005;112(8):1394.

3. Cornea Donor Study Investigator Group, Gal RL, Dontchev M, Beck RW, Mannis MJ, Holland EJ, Kollman C, et al. The effect of donor age on corneal transplantation outcome results of the cornea donor study. Ophthalmology. 2008;115(4):620-626.e6.

4. Cornea Donor Study Investigator Group, Lass JH, Gal RL, Dontchev M, Beck RW, Kollman C, Dunn SP, et al. Donor age and corneal endothelial cell loss 5 years after succesful corneal transplantation. Specular microscopy ancillary study results. Ophthalmology. 2008;115(4):627-632.e8.

5. Williams KA, Muehlberg SM, Lewis RF, Coster DJ. Influence of advanced recipient and donor age on the outcome of corneal transplantation. Australian Corneal Graft Registry. Br J Ophthalmol. 1997;81(10):835-9. Comment in: $\mathrm{Br}$ J Ophthalmol. 1997;81(10):809-10.

6. Eye Bank Association of America. Medical Standards. Washington, DC: Eye Bank Association of America; 1993.

7. Gain P, Rizzi P, Thuret G, Chiquet C, Michel-Valanconny C, Pugniet JL, et al. Prélèvements de cornées après 85 ans: issue des greffons après organoculture et devenir des greffés. J Fr Ophtalmol. 2002;25(3):274-89.

8. Georgiadis N, Kardasopoulos A, Bufidis T. The evaluation of corneal graft tissue by the use of trypan blue. Ophthalmologica. 1999;213(1):8-11.

9. Krohn J, Hovding G. The influence of donor age and cause of death on corneal endothelial cell density. Acta Ophthalmol Scand. 2005;83(6):746-50. 